\title{
Mucopiocele fronto-orbitária e transtorno comportamental: relato de caso
}

\author{
Leonardo C. Welling ${ }^{1}$, José Carlos Lynch², Celestino E. Pereira ${ }^{3}$, Leandro A. Ferreira ${ }^{4}$ \\ Hospital dos Servidores do Estado do Rio de Janeiro (HSE/RJ)
}

RESUMO

Objetivo: Descrever uma patologia rara, de crescimento lento, com bom prognóstico se adequadamente tratada. Método: Análise e descrição de um caso de mucopiocele fronto-orbitária e transtorno comportamental, submetido ao tratamento cirúrgico no Hospital dos Servidores do Estado do Rio de Janeiro. Resultados: O paciente evoluiu com regressão completa dos sintomas prévios. Conclusão: Nas mucopioceles, os objetivos da cirurgia são: a confirmação do diagnóstico, a drenagem do conteúdo de muco e pus e a excisão ou marsupialização das paredes do cisto e da mucosa do seio comprometido. O diagnóstico correto dessa lesão leva à diminuição da morbidade e mortalidade, em virtude de o tratamento cirúrgico associado à antibioticoterapia venosa ser curativo.

\section{PALAVRAS-CHAVE}

Mucopiocele, intracraniana, transtorno comportamental.

\begin{abstract}
Intracranial mucopyocele and cognitive impairment: case report

Objective: To describe an uncommon pathology, slow-growing and good prognosis if well treated. Method: Analyze and description of fronto-orbital mucopyocele associated with cognitive impairment submitted a surgical treatment in Hospital dos Servidores do Estado do Rio de Janeiro. Results: Following the surgery all previous symptoms resolved. Conclusion: The objectives of surgery in mucopyoceles are diagnostic confirmation, drainage of secretion, excision or marsupialization of cyst walls and mucous of compromised sinus. The correct diagnosis reduces de morbidity and mortality since the surgical treatment associated with venous antibiotics is curative.
\end{abstract}

\section{KEYWORDS}

Mucopyocele, intracranial, cognitive impairment.

\section{Introdução}

As mucoceles caracterizam-se como processos expansivos benignos e de crescimento lento, preenchidos por muco e revestidos por epitélio respiratório, localizados nos seios paranasais. A obstrução dos óstios de drenagem desses seios é um fator predisponente para a formação das mucoceles, podendo estas ser tão extensas a ponto de causar invasão e compressão das estruturas orbitárias e/ou intracranianas. ${ }^{5}$ Quando se tornam infectadas, são denominadas mucopioceles.?

As localizações mais frequentes são os seios frontal, seguidos pelo etmoide, maxilar e esfenoide. ${ }^{4}$ Os sintomas mais frequentes são cefaleia, rinorreia e obstrução nasal. O diagnóstico por imagem é feito por meio da tomografia computadorizada de crânio (TCC) e seios da face ou com ressonância magnética do encéfalo (RMe).

O objetivo da cirurgia é drenar a coleção mucopurulenta e eliminar o efeito de massa. A abordagem cirúrgica varia conforme a localização da lesão. Exposições faciais e transcranianas são realizadas. O tratamento cirúrgico é complementado com antibioticoterapia venosa.

\section{Relato do caso}

JFC, 55 anos, masculino, natural da Bahia, com história de três meses de evolução com cefaleia, proptose à direita, transtorno comportamental e sinusopatia crô-

1 Neurocirurgião, ex-residente do Serviço de Neurocirurgia do HSE/RJ, doutorando do Programa de Pós-Graduação da Universidade de São Paulo (USP).

2. Neurocirurgião, chefe do Serviço de Neurocirurgia do HSE/RJ.

3. Neurocirurgião, preceptor dos residentes do HSE/RJ.

4. Neurocirurgião, ex-residente do Serviço de Neurocirurgia do HSE/RJ. 
nica. TCC de crânio evidenciou extensa coleção frontal direita captante de contraste e contígua com sinusopatia em seio etmoide (Figuras 1 e 2).

O paciente foi submetido à craniotomia frontal direita e na trepanação do ptério houve drenagem de grande quantidade de muco e pus (Figura 3). A craniotomia foi completada, a secreção epidural foi totalmente drenada e removeu-se a cápsula da lesão. A cavidade do seio esfenoidal foi curetada e a mucosa remanescente, removida. A lesão era extradural, ocupando cavidade intracraniana frontal direita e parcialmente à esquerda, além de destruir a órbita direita. Não se identificou extensão intradural.

Houve importante melhora clínica na primeira semana pós-operatória, com reexpansão do lobo frontal direito (Figura 4). No material, evidenciou-se o crescimento de germes da família Corynebacterium.

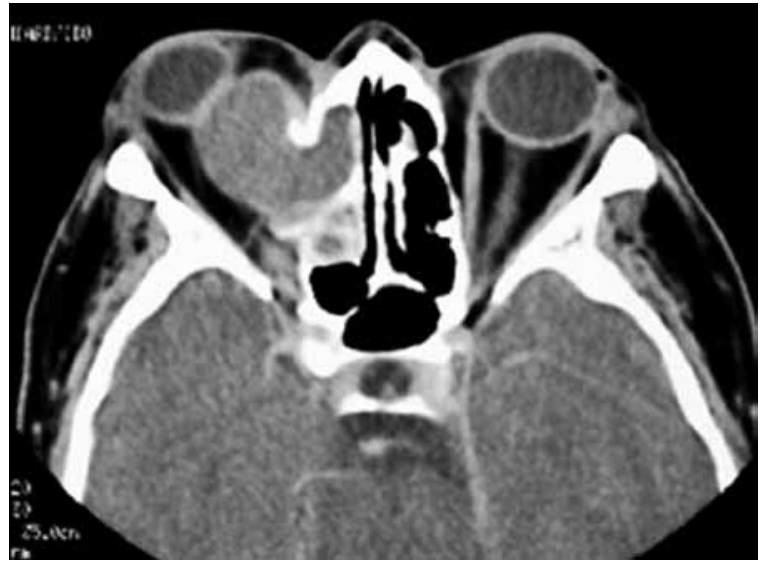

Figura 1 - Corte axial. Observam-se coleção isodensa nos seios etmoides à direita, invasão da órbita e deslocamento do globo ocular.

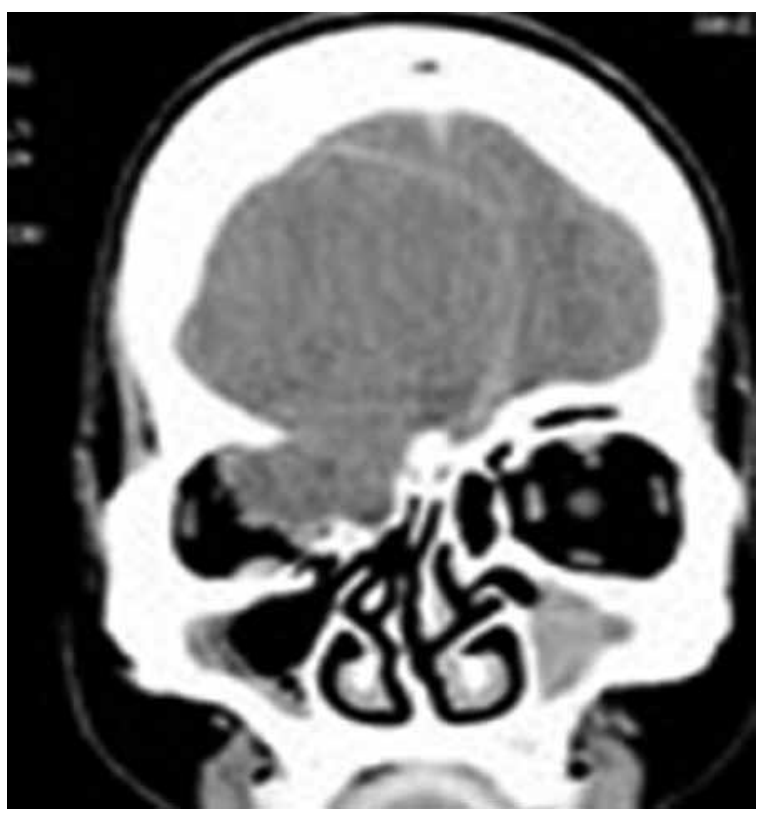

Figura 2 - TC em corte coronal mostra erosão do teto da órbita e invasão intracraniana.
O paciente foi submetido à antibioticoterapia (cefepime, vancomicina e metronidazol). O tratamento estendeu-se por seis semanas.

Durante seu acompanhamento ambulatorial, foram observadas completa regressão dos sintomas psicóticos e regressão da proptose do olho direito, e o paciente retornou às suas atividades prévias.

\section{Discussão}

As mucoceles, e principalmente as mucopioceles, apesar de serem lesões benignas, têm grande potencial de destruição óssea e invasão das estruturas adjacentes.

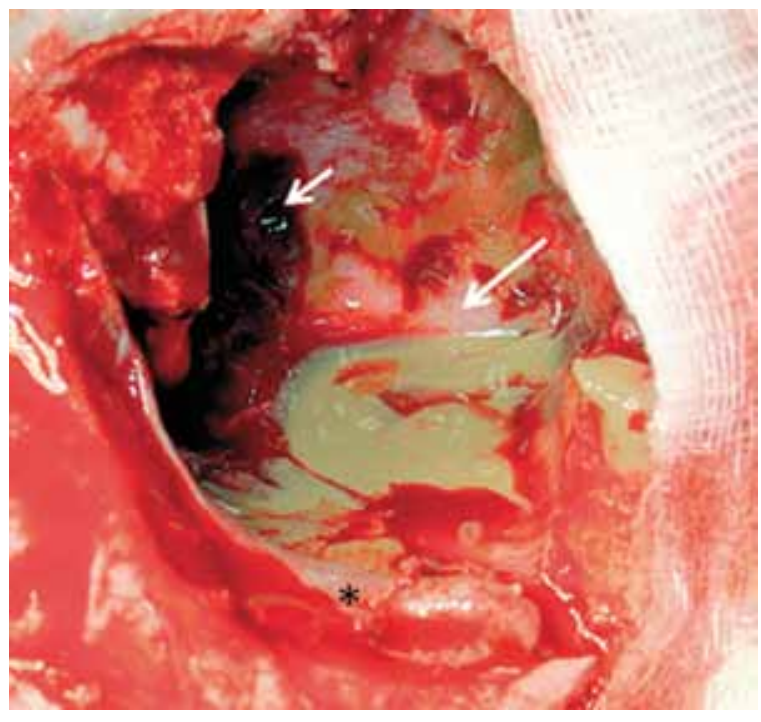

Figura 3 - Craniotomia frontal direita com material purulento na cavidade. Observam-se seio etmoidal parcialmente destruído (seta curta), teto da órbita (seta longa) e cápsula da lesão (asterisco).

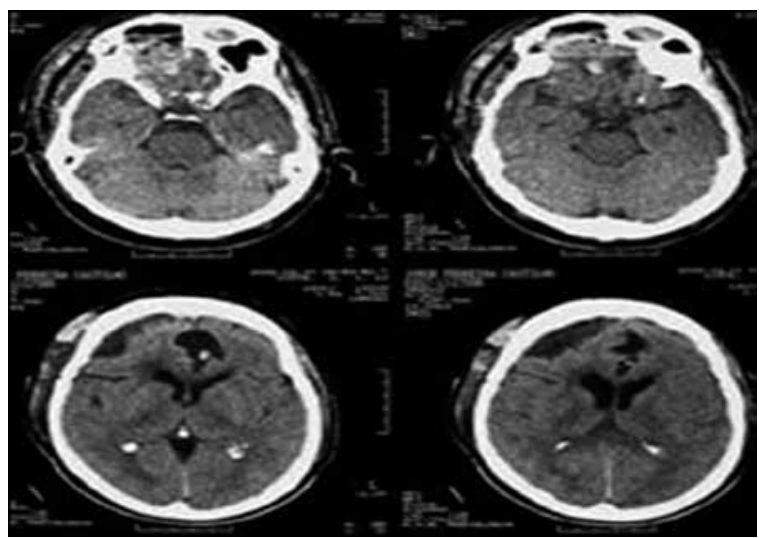

Figura 4 - Controle pós-operatório na primeira semana. 
O aumento gradual do volume da lesão leva à isquemia e à liberação de enzimas proteolíticas e mediadores enzimáticos, que resultam em destruição óssea e expansão da mucopiocele. ${ }^{6}$

A maioria das mucoceles é diagnosticada em adultos, podendo ocorrer em qualquer faixa etária. Não se observa predominância de sexo. ${ }^{6}$ Algumas vezes, as mucopioceles são diagnosticadas por meio de tumoração subcutânea localizada na região frontal. ${ }^{1}$

A TCC de crânio mostra dilatação da cavidade do seio paranasal comprometido, com adelgaçamento das paredes ósseas, porém geralmente íntegras. A mucocele apresenta características homogêneas e hipodensas, com fraca captação periférica do contraste. ${ }^{10} \mathrm{~A}$ mucosa de revestimento dos seios perde as suas características histológicas, desaparecendo a camada ciliar, formando-se um verdadeiro cisto. ${ }^{6}$

O diagnóstico diferencial é com a displasia fibrosa, fibroma ossificante e outras neoplasias. ${ }^{6}$

Na literatura, há relatos da associação de mucoceles com trauma craniano prévio, ${ }^{13}$ complicações pós-operatórias levando à obstrução da drenagem do respectivo seio, ${ }^{15}$ sinusopatia crônica levando à hipersecreção mucosa. ${ }^{8}$ Existe a associação de mucoceles frontais com osteomas. ${ }^{3} \mathrm{~A}$ flora da mucopiocele é composta por bactérias aeróbias e anaeróbias. Segundo Brook e Frazier, ${ }^{2}$ dos germes aeróbios, o Staphylococcus e o Streptococcus são os mais frequentes e, dentre os anaeróbios, os Peptostreptococcus sp.

Uma manifestação característica e que esteve presente nesse caso é a presença de cefaleia e proptose quando ocorre invasão das estruturas intraorbitárias. ${ }^{10}$ Quando ocorre invasão da dura-máter, a primeira manifestação pode ser uma meningite e/ou crises convulsivas ${ }^{9}$, ou até mesmo um abscesso cerebral. ${ }^{11}$ No nosso caso, o que mais chamou a atenção foi o transtorno comportamental à custa da grande compressão exercida pela massa nos lobos frontais.

Existem várias teorias que explicam a origem das mucoceles, além da pura e simples obstrução dos óstios de drenagem. ${ }^{8}$ Segundo Tamas e Wyler, pode existir um pequeno seio ectópico, não visualizado pelos métodos de imagem, ou até mesmo uma conexão com o seio frontal que desaparece no início da vida dos pacientes. ${ }^{14}$

\section{Conclusão}

As mucoceles e mucopioceles raramente invadem as estruturas intracranianas. Os objetivos da cirurgia são a confirmação do diagnóstico, a drenagem do conteúdo de muco e pus e a excisão ou marsupialização das paredes do cisto e da mucosa do seio comprometido.
Deve ser enfatizada a importância do diagnóstico dessa lesão em virtude de o tratamento cirúrgico associado à antibioticoterapia venosa ser curativo, o que leva à diminuição da morbidade e mortalidade.

Portanto, deve-se incluir no diagnóstico diferencial a presença de mucocele ou mucopiocele nas lesões com invasão intracraniana, principalmente naquelas associadas à sinusopatia crônica.

\section{Referências}

1. Akiama M, Inamoto N, Hashigucci K. Frontal mucocele presenting as a subcutaneous tumor on the forehead. Dermatology. 1999;199:263-4.

2. Brook I, Frazier EH. The microbiology of mucopyocele. Laryngoscope. 2001;111(10):1771-3.

3. Brunori A, Bruni P, Delitala A, Greco R, Chiappetta F. Frontoetmoidal osteoma complicated by intracranial mucocele and hypertensive pneumocephalus: case report. Neurosurgery. 1995;36:1237-8.

4. Curtin HD, Rabinov JD. Extension to the orbit from paraorbital disease: the sinuses. Radiol Clin North Am. 1998;36(6):1201-13.

5. Delfini R, Missori P, lannetti G, Ciappetta P, Cantore G. Mucoceles of the paranasal sinuses with intracranial and intraorbital extension: report of 28 cases. Neurosurgery. 1993;32(6):901-6.

6. Galie M, Mandrioli S, Tieghi R, Clauser L. Giant mucocele of the frontal sinus. J Craniofac Surg. 2005;16(5):933-5.

7. Hashim AS, Asakura T, Awa H, Yamashita K, Takasaki K, Yuhi F. Giant mucocele of paranasal sinuses. Surg Neurol. 1985;23(1):69-74.

8. Kpemissi E, Balo K, Kpdozro K. Mucoceles sinusiennes: à propos de observations. Ann Otolaryngol Cervicofac. 1996;11:179-82.

9. Nabeshima K, Marutsuka K, Shimao Y, Uehara H, Kodama K. Osteoma of the frontal sinus complicated by intracranial mucocele. Pathol Int. 2003;53:227-30.

10. Nakayama T, Mori K, Maeda M. Giant pyocele in the anterior intracranial fossa: case report. Neurol Med Chir (Tokyo). 1998;38:499-502.

11. Perie S, Sequert C, Cabanes J, Visot A, Derome P. Mucoceles frontales à extension orbitaire ou cerebrale: strategie therapeutique. Ann Otolaryngol Cervicofac. 1996;113:384-91.

12. Selvapandian S, Rajshekhar V, Chandy MJ. Mucoceles: a neurosurgical perspective. Br J Neurosurg. 1994;8(1):57-61.

13. Smoot EC, Bowen DG, Lappert P, Ruiz JA. Delayed development of an ectopic frontal sinus mucocele after pediatric cranial trauma. J Craniofac Surg. 1995;6:327-31.

14. Tamas LB, Wyler AR. Intracranial mucocele mimicking arachnoid cyst: case report. Neurosurgery. 1985;16:85-6.

15. Wang L, Kim J, Hallman CB. Intracranial mucocele as a complication of endoscopic repair of cerebrospinal fluid rhinorrhea: case report. Neurosurgery. 1999;45:1243-6.

Endereço para correspondência

Leonardo C. Welling

Rua Tiradentes, 976, Centro

84010-190 - Ponta Grossa, PR

E-mail: leonardowelling@yahoo.com.br 